\title{
Percepciones lingüísticas de hablantes bilingües: análisis evaluativo
}

\author{
Linguistic Perceptions of Bilingual Speakers: \\ an evaluation analysis \\ Teresa Oteíza \\ Universidad Austral de Chile, Instituto de Lingüística y Literatura, Valdivia, Chile. \\ e-mail: teresaoteiza@uach.cl
}

\begin{abstract}
En este estudio se analizan las percepciones lingüísticas de un grupo de hablantes bilingües universitarios que viven en Estados Unidos y cuya lengua materna/heredada es el español. El análisis de las evaluaciones de su competencia en español, tanto en registros coloquiales como académicos, se ha realizado desde un Análisis de Evaluación y la Lingüística Sistémico Funcional. En conjunto, estos modelos ofrecen un marco teórico y metodológico que permite examinar significados interpersonales tanto a nivel léxico-gramatical como discursivo-semántico. La comprensión de las subjetividades desde donde se sitúan estos hablantes bilingües, en proceso de adquirir un español formal que les permita desenvolverse con éxito en otros contextos que no sean las interacciones coloquiales, resulta aún más crítica si se tiene en cuenta que el español es una lengua minoritaria en el país.
\end{abstract}

Palabras clave: análisis de evaluación, hablantes de español como lengua materna/heredada, percepciones lingüísticas, lingüística sistémico funcional.

In this study we analyze the language perceptions of a group of college bilingual speakers who live in the United States whose native/heritage language is Spanish. The evaluation analysis of their proficiency in Spanish, both in colloquial and academic registers, has been done from an Appraisal Analysis and Systemic Functional Linguistics. Together, these models provide a theoretical and methodological framework that allows us to examine interpersonal meanings at the lexico-grammatical and discourse-semantic level. An adequate understanding of the subjectivities from where bilingual speakers are situated, while in the process of acquiring formal Spanish to successfully perform in contexts other than colloquial interactions, appears even more crucial if we take into consideration that Spanish is a minority language in the country.

Key words: appraisal analysis, Spanish heritage speakers, language perceptions, minority languages, systemic functional linguistics.

\section{INTRODUCCIÓN}

Este estudio explora las valoraciones lingüísticas acerca del español en un grupo de hablantes bilingües de Estados Unidos cuya lengua materna/heredada es el español. Se han analizado sus percepciones en relación a su capacidad para expresarse en español tanto en registros coloquiales como en registros académicos en sus modalidades orales y escritas. Este interés surge como parte de una investigación más amplia en torno al proceso de adquisición y desarrollo de un español académico en hablantes bilingües que viven en los Estados Unidos. Para realizar este estudio se ha empleado un Análisis de Evaluación o Appraisal Analysis (Martin 2000; Hunston y Thompson 2000; Martin y Rose 2003; Martin y White 2005) y la Lingüística Sistémico Funcional (Halliday 1994, 2001; Eggins 1994), dado que son modelos teóricos que permiten dar cuenta de las actitudes manifestadas por los hablantes y su expresión a nivel léxicogramatical y discursivo-semántica.

En trabajos anteriores (Oteíza en prensa) se ha analizado el proceso de adquisición de un español académico escrito en este mismo grupo de hablantes bilingües que permita entregar una descripción de los recursos lingüísticos que ellos utilizan para superar la limitada exposición y formación que tienen en estos registros de la lengua. En un contexto de educación universitaria, los hablantes bilingües (HBs) se ven en la necesidad de emplear géneros de tipo argumentativo y expositivo en español con escasa o nula experiencia previa en el uso de este tipo de lenguaje. Frente a este desafío, el lenguaje típicamente empleado descansa en patrones lingüísticos de un español hablado y coloquial con el que tienen mayor familiaridad.

Si bien en Estados Unidos, especialmente a partir de los años setenta, comienza a haber una preocupación en relación al desarrollo y mantención del español entre hablantes bilingües y se aprecia un 
aumento en el número de clases y materiales diseñados especialmente para esta población minoritaria en el país, sólo algunos estudios se han enfocado en las percepciones que tienen los hablantes bilingües de inglés-español enfrentados al proceso de adquisición de registros más formales en español (Potowki 2002; Beckstead y Toribio 2003; Carrasco y Riegelhaupt 2003; Castillo 2003, entre otros). Beckstead y Toribio, por ejemplo, investigaron las actitudes hacia el inglés y español en un grupo de adolescentes mexicanos y méxico-americanos. Estos autores concluyeron que los adolescentes que participaron en el estudio manifestaban una valoración positiva tanto por el inglés como por el español, a la vez que reconocían el valor del español como una forma de preservar su identidad latina. Estos estudiantes, además, manifestaron que si bien apreciaban el valor instrumental del inglés, preferían el uso del español durante las clases. Por su parte, Carrasco y Riegelhaupt, en el contexto del desarrollo de un modelo que tiene por objeto la continuación de la adquisición del español entre hablantes bilingües de inglés-español indagaron sobre las expectativas que tenían, desde un punto de vista social y de ideologías lingüísticas, tanto estudiantes y futuros profesores de español y las familias que los recibían en México como parte de un período de formación. Sin embargo, no se ha realizado un estudio lingüístico que analice los testimonios de los HBs en un contexto universitario y explore la expresión de actitudes de evaluación y sus manifestaciones a nivel léxico-gramatical y discursivo-semántico. Normalmente, se asumen las necesidades que estos grupos de hablantes requieren para adquirir y mantener una alfabetización avanzada en español, sin considerar la subjetividad desde donde se sitúan los propios involucrados en este proceso.

El concepto de registros académicos guarda relación con el lenguaje usado en instituciones educacionales. En el contexto de determinadas prácticas sociales el lenguaje es empleado de acuerdo a ciertas demandas lingüísticas y de estructuración del lenguaje con las que los estudiantes no están necesariamente familiarizados. Los registros académicos han sido descritos ampliamente de acuerdo al marco teórico de la Lingüística Sistémico Funcional (LSF) (Eggins 1994; Eggins, Martin 1997; Christie 1999; Halliday 1994, 2001; Colombi, Schleppegrell 2002, Schleppegrell 2004, entre otros) la cual considera la alfabetización avanzada (literacy) como una actividad semiótica social. De esta manera, la noción de registro, según LSF es crucial para entender cómo el contexto -que se construye socialmentese realiza en el lenguaje como una actividad lingüística. Como una forma de acción social, las variedades académicas escritas de una lengua difieren en varios rasgos lingüísticos de las variedades conversacionales del lenguaje cotidiano. Esto es, cada registro de lenguaje posee un contexto particular que se realiza a través de distintos recursos lingüísticos. Aunque existen investigaciones que han descrito diferentes variedades orales y coloquiales del español de Estados Unidos (Lispki 2000; Silva-Corvalán 1994, 2001, entre otros), sólo hasta en los últimos años se le ha prestado atención a la adquisición de registros académicos de la lengua tanto oral como escrita en contextos académicos (Valdés and Geoffrion-Vinci 1998, Colombi 2000, 2003; Achugar 2003; Acevedo 2003; Oteíza (en prensa).

Nuestro trabajo como educadores y lingüistas con este grupo de hablantes se ve influenciado, preferentemente, por los discursos públicos e institucionales que nos informan sobre las necesidades y formas de desarrollar una alfabetización avanzada en una comunidad de habla hispana que está en rápido y continuo crecimiento en Estados Unidos; sin embargo, en este estudio no se trabaja con tales discursos sino con discursos privados o personales de un grupo de hablantes de una comunidad específica. Se considera que un estudio cualitativo de esta naturaleza puede colaborar a entender mejor a una comunidad de habla que tradicionalmente ha sido marginada en su educación formal de la lengua materna/heredada de sus padres.

¿Cuáles son sus percepciones en relación al español?, ¿cómo evalúan su propia proficiencia en diferentes registros y contextos de uso?, ¿cómo evalúan sus posibilidades de acceso a otros espacios de participación en la sociedad estadounidense? Estas son algunas de las interrogantes de investigación que se desean explorar en este estudio.

\section{PARTICIPANTES Y RECOLECCIÓN DE DATA}

En este estudio participaron $30 \mathrm{HBs}$ que asisten a una universidad de Washington en pregrado. Sus edades fluctúan entre 18 a 25 años. Veintidós de los participantes (73,3\%) han vivido toda su vida en Estados Unidos, específicamente en pueblos pequeños del área central del estado de Washington. Ellos pertenecen a una primera y segunda generación de hablantes de español. El grupo restante de 12 participantes (40\%) nació en países de habla hispana en la siguiente distribución: diez de ellos en México, uno en El Salvador y uno en Venezuela, y llegaron a Estados Unidos a una edad temprana (antes de los seis años). Sólo dos de ellos completaron su educación primaria antes de salir de sus países. Los padres de 
los participantes de este estudio, con excepción de dos, son trabajadores del campo. Todos los padres son hablantes de español como lengua materna con excepción de tres participantes que tienen sólo un padre o una madre que habla español como primera lengua.

De los 30 participantes, 27 de ellos corresponden a la primera generación en sus familias que tienen acceso a una educación universitaria. La mayoría habla dialectos rurales del español aprendido de sus padres y/o parientes que han emigrado a Estados Unidos desde pueblos pequeños de sus respectivos países. La mayoría no utiliza el español fuera de las clases y sólo lo emplean para hablar en ambientes familiares y con amigos. Muchos de ellos manifiestan que tienen pocas oportunidades de practicar su español cuando están en la universidad y que sólo lo usan cuando regresan a sus casas en los periodos de vacaciones o cuando se comunican con sus padres y algunos amigos.

Es importante tener en cuenta que los participantes forman un grupo heterogéneo en relación a los años que cada uno ha recibido de instrucción formal en español dentro de Estados Unidos. Trece de ellos han recibido dos años o menos de instrucción formal en esta lengua en la educación secundaria (junior high y high school), y nueve han recibido entre tres a cinco años de clases de español en Estados Unidos. Las clases de español a las que asistieron estaban dirigidas principalmente a los estudiantes que desean aprender español como segunda lengua y, por lo mismo, tanto la metodología como los materiales empleados no estaban adecuados a hablantes de español como lengua hereditaria. Más de la mitad de los participantes que corresponde al 60 por ciento de la muestra considera que el inglés en su lengua dominante, en especial si consideran su escritura. El 26,6 por ciento considera que ambas lenguas se encuentran a un mismo nivel de proficiencia, y un 13,3 por ciento piensa que son más competentes en español.

Todos los participantes contestaron un cuestionario preliminar en el que entregaron información personal general, datos de su historia de socialización en Estados Unidos y de sus patrones de uso del español y del inglés. Luego entregaron por escrito un párrafo de no más de 20-25 líneas sobre su experiencia con el español en sus vidas. Este párrafo lo podían escribir con el formato que quisieran y se entregaba, al igual que el cuestionario preliminar, vía correo electrónico a la investigadora. A continuación se les solicitó que contestaran un cuestionario de autopercepción sobre su uso del español (ver Anexo). 16 de los 30 participantes fueron además entrevistados personalmente por la investigadora como una manera de contrastar, confirmar y completar la información entregada por los instrumentos previamente señalados.

El hecho de que la fuente principal de este estudio lo constituyan el testimonio escrito y las respuestas escritas de los participantes tiene consecuencias en el momento de analizar los resultados obtenidos. Los participantes idealmente tuvieron tiempo para pensar, escribir y corregir, si así lo requerían, toda la información pedida. También es relevante el hecho de que además la investigadora haya sido en diferentes momentos profesora de lengua de los participantes del estudio. Esto claramente puede haber influido en sus repuestas, si bien como parte de las instrucciones dadas en reuniones previas con todos ellos se les enfatizó que el propósito del estudio era conocer sus percepciones sobre la lengua y sus capacidades, y que no serían evaluados por su redacción, ortografía o uso del lenguaje en general. Los participantes tuvieron, entonces, desde el principio conciencia de la dirección de la investigación y se les aseguró que sus datos permanecerían en el anonimato. El 80 por ciento de estos participantes ya había participado en un estudio previo relacionado con el proceso de adquisición de registros escritos del español académico.

Los resultados que presento en este artículo son producto de un análisis evaluativo del párrafo de testimonio de los HBs, así como de las respuestas del cuestionario de percepciones lingüísticas entregadas por ellos.

3. Metodología

Los datos recogidos fueron analizados de acuerdo a un Análisis de Evaluación (AE) que es desarrollado por Martin y Rose (2003); Martin y White (2005), entre otros, en el marco de la Lingüística Sistémico Funcional (LSF). Este análisis ofrece herramientas analíticas para explorar los significados interpersonales que se construyen en el discurso. La flexibilidad que este tipo de análisis posibilita se debe, en parte, al principio de que ningún elemento por sí solo genera este tipo de significados sino que estos significados, interpersonales serán construidos por la combinación de realizaciones gramaticales como léxicas en el discurso. En nuestros discursos los hablantes/escritores comunicamos a nuestros oyentes/lectores cómo pensamos y sentimos acerca tanto de cosas, eventos y gente; es decir, comunicamos cuáles son nuestras actitudes. De acuerdo con Hunston y Thompson (2000), la evaluación es importante porque desempeña un rol crucial en la construcción de los fundamentos ideológicos del discurso y, por lo mismo, es posible situar al lector y autor en un espacio 
ideológico que nos permita entender el sistema de valores de esa persona y su comunidad. La evaluación es a su vez relevante ya que ayuda a mantener una relación entre hablante/autor y oyente/lector y además porque juega un papel importante en la organización del discurso, es decir, en cómo el argumento es construido en el discurso. Martin y Rose (2003) sugieren que habrían tres sistemas evaluativos principales: Actitud, Compromiso y Graduación. A continuación se explica el significado y función de cada uno de estos sistemas y cómo se llevó a cabo el análisis.

3.1. El Sistema de Actitud puede subdividirse en los subsistemas de Afecto, Juicio y Apreciación. Cada uno puede ser positivo, negativo o ambos, además de que estas evaluaciones pueden ser expresadas directa e indirectamente en el discurso. (i) El subsistema de Afecto guarda relación con la expresión de nuestros sentimientos frente a la cosa, persona o evento evaluado. Estos pueden ser a grandes rasgos de Felicidad, Seguridad y Satisfacción o Infelicidad, Inseguridad, Insatisfacción. (ii) El susbsistema de Juicio tiene relación con la institucionalización de los sentimientos, es decir, se refiere a cómo la gente debiera o no debiera comportarse en una comunidad determinada. Este puede expresarse a nivel de Estima Social como de Sanción Social en relación al comportamiento humano y las normas sociales que lo gobiernan en determinadas comunidades. Martin y Rose (2003) consideran las categorías de Normalidad, Capacidad y Tenacidad para el análisis de personas. La Sanción Social, en cambio se asocia a las evaluaciones de lo considerado "bueno" o "malo", por lo tanto, dentro de esta subclasificación cabrían categorías como Veracidad (verdad, honestidad) y Propiedad o Corrección (moral, ético, justo). Volveré sobre estas categorías más adelante para discutir su adecuación en el estudio de las percepciones lingüísticas. (iii) El subsistema de Apreciación guarda relación con la evaluación que asignamos a las cosas o fenómenos naturales; esta evaluación puede ser en términos de su composición, reacción o valor.

3.2. El Sistema de Compromiso se refiere a la(s) fuente(s) de las actitudes o a las personas que evalúan. Este aspecto del análisis toma en consideración de dónde proviene la evaluación. "Esta potencial existencia de varias fuentes de lo que es dicho es uno de los factores que llevaron, Bakhtin (1981) a pensar sobre la naturaleza dialógica del discurso, incluso en textos que tradicionalmente son considerados monólogos" (Martin \& Rose 2003:44). Será la analista francesa del discurso, Kristeva quien introducirá el término "heteroglosia" para esta noción de la multiplicidad de voces que están presentes en todo discurso. En este estudio se toma en consideración esta noción de la manera en que la entienden Martin y Rose; esto es, cuando la fuente de una actitud es otra diferente a la del(a) autor(a), o dicho de otro modo, diferente de la voz del(a) HB, la considero heteroglosia, y monoglosia ('una sola voz') cuando la fuente es el(a) autor(a), es decir él o la HB. La heteroglosia puede ser codificada en el discurso a través de proyecciones mentales y verbales (cláusulas proyectadas en el discurso). Otra posibilidad es introducir voces adicionales vía empleo de la modalidad (Halliday 1994). En la presente data se ha considerado la modalidad en términos de negociación de información, esto es, "cuán probable" es una afirmación desde el punto de vista de cómo es planteada por quién la expresa. La modalidad, de esta manera, es usada en este análisis como una gradación de posibilidades y habilidades que fluctúan entre un polo positivo y negativo. Esta categoría está conectada a la evaluación de la capacidad de usar español en diferentes registros por parte de los HBs. Como señala Halliday, la modalidad, así como la polaridad, reconoce voces alternativas en torno a una sugerencia o afirmación. Si bien la modalidad, "a diferencia de la polaridad, no toma esas voces para luego negarlas, sino que más bien abre un espacio de negociación en el que diferentes puntos de vista pueden circular en torno al asunto, un espacio tal vez para una mediación y posible reconciliación" (1994: 50).

La categoría de Concesión se relaciona con la forma en que el hablante/autor monitorea las expectativas del oyente/lector. Así, por ejemplo, el uso de conectores o marcadores discursivos de carácter adversativos del tipo "pero" y "aunque" (cuando son usados con este valor) señalan una expectativa contraria que el hablante/autor ha creado para el oyente/lector.

3.3. El Sistema de Graduación o amplificación se refiere a un rasgo distintivo de las actitudes: pueden ser graduables. Por consiguiente, las evaluaciones pueden ser más o menos intensas en la medida en que son más o menos amplificadas (Martin y Rose 2003). De acuerdo con esta característica es posible decir cuán firmemente sentimos y pensamos acerca de alguien o algo. De esta manera, por ejemplo se establece una diferencia cuando un(a) HB sostiene que: "Realmente pienso que mi español hablado en muy bueno" expresando con firmeza su proficiencia oral y coloquial, en contraste con el/la que afirma que "mi español hablado es bueno" sin amplificar el léxico implicado en la expresión de su punto de vista. En el primer ejemplo el adjunto comentario "Realmente" le otorga más fuerza a la aseveración ya 
que afecta a toda la cláusula proyectada por el verbo mental. Es posible, entonces, intensificar nuestros significados (fuerza) como lo expresa el ejemplo; o podemos enfatizar o suavizar nuestras evaluaciones (foco) con palabras del tipo "exactamente", "como", "del tipo de", etc., acotando o delimitando su significado. En suma, "la ausencia de graduación colabora a mantener las definiciones más nítidas y precisas lo mismo que ocurre con la ausencia de proyección, modalización y concesión que colaboran en reforzar un punto de vista monovocal" (Cf. 2003:55).

A continuación, en la tabla 1, se presenta una ejemplificación de cómo han sido tratados cada uno de los aspectos del $\mathrm{AE}^{1}$.

Tabla 1

Ejemplos de análisis

\begin{tabular}{|c|c|c|c|c|}
\hline $\begin{array}{l}\text { Quién o qué es } \\
\text { evaluado } \\
\text { Actor social } \\
\text { Cosa } \\
\text { Evento }\end{array}$ & $\begin{array}{l}\text { Discurso } \\
\text { (palabras } \\
\text { textuales) }\end{array}$ & $\begin{array}{l}\text { Actitud: } \\
\text { (a)Afecto } \\
\text { (b) Juicio: } \\
\text { Estima Social } \\
\text { Sanción Social } \\
\text { (c) Apreciación }\end{array}$ & $\begin{array}{l}\text { Compromiso } \\
\text { (Fuentes de las } \\
\text { actitudes o } \\
\text { persona que } \\
\text { evalúa) } \\
\text { (Monoglosia, } \\
\text { heteroglosia, } \\
\text { proyección, } \\
\text { modalidad, } \\
\text { concesión) }\end{array}$ & Graduación: \\
\hline $\begin{array}{l}\text { (1) Proficiencia } \\
\text { oral } \\
\text { (informal con la } \\
\text { familia) }\end{array}$ & $\begin{array}{l}\text { Puedo hablar } \\
\text { claramente } \\
\text { aunque sin } \\
\text { duda hay } \\
\text { algunas } \\
\text { veces que sé } \\
\text { que aunque } \\
\text { nos } \\
\text { entendemos no } \\
\text { estamos } \\
\text { hablando } \\
\text { correctamente } \\
\text {. }\end{array}$ & $\begin{array}{l}\text { Juicio de } \\
\text { Estima social } \\
\text { positiva: } \\
\text { +capacidad } \\
\text { Juicio de } \\
\text { Estima social } \\
\text { negativa: } \\
\text {-capacidad } \\
\text { Apreciación: } \\
\text { - corrección }\end{array}$ & $\begin{array}{l}\text { Monoglosia: HB } \\
\text { Modalidad } \\
\text { positiva: } \\
\text { puedo hablar } \\
\text { claramente } \\
\text { aunque sin } \\
\text { duda } \\
\text { Polaridad } \\
\text { negativa: } \\
\text { no estamos } \\
\text { hablando } \\
\text { correctamente. } \\
\text { Concesión: } \\
\text { aunque } \\
\text { aunque }\end{array}$ & $\begin{array}{l}\text { Foco: } \\
\text { claramente } \\
\text { sin duda } \\
\text { algunas } \\
\text { veces } \\
\text { correctament } \\
\text { e }\end{array}$ \\
\hline $\begin{array}{l}\text { (2) Español } \\
\text { formal oral } \\
\text { (presentación } \\
\text { formal en la sala } \\
\text { de clases) }\end{array}$ & $\begin{array}{l}\text { Creo que todos } \\
\text { estarían de } \\
\text { acuerdo } \\
\text { conmigo de } \\
\text { pensar/decir } \\
\text { que mi español } \\
\text { es a un nivel } \\
\text { más o menos. }\end{array}$ & $\begin{array}{l}\text { Juicio de } \\
\text { Estima social: } \\
\text { +/-capacidad }\end{array}$ & $\begin{array}{l}\text { Heteroglosia } \\
\text { Proyección } \\
\text { mental y verbal } \\
\text { todos/ voz del(a) } \\
\text { HB }\end{array}$ & $\begin{array}{l}\text { Fuerza: } \\
\text { Grado medio } \\
\text { más o menos }\end{array}$ \\
\hline
\end{tabular}

Con anterioridad a la presentación del análisis parece relevante precisar de qué manera se está considerando la categoría de "Corrección" en el análisis y por qué ha sido incluida bajo el subsistema de Apreciación, no sin ciertas reservas. En el análisis del lenguaje y de la proficiencia que un hablante puede

\footnotetext{
${ }^{1}$ En el análisis se ha respetado la puntuación, ortografía, redacción e inclusión de palabras en inglés de cada uno de los informantes del estudio.
} 
expresar de su uso del lenguaje, la expresión de que "mi español no es correcto" o de que "debo aprender a hablar más correctamente/educadamente" responde a una valoración de Apreciación; sin embargo, no se podría emplear en los términos que Martin y Rose utilizan esta subcategoría de valoración, es decir, como "desafiante, significativo, profundo, provocativo..." y sus significados opuestos (Cf. 2003: 63). Se considera que cuando un(a) HB se refiere a la "corrección del idioma" se está refiriendo en parte a la categoría de composición ("balanceado, armonioso, simple, elegante, preciso"... y sus opuestos), pero pareciera que lleva otro significado añadido: un carácter de "bueno" o "malo" y del "deber ser" de la lengua. Ahora bien, pensar que están expresando un Juicio de Sanción Moral de propiedad: "bueno, moral, ético, justo" y sus opuestos, parece más apropiado para evaluar personas individuales o colectivos, pero no la competencia lingüística del(a) HB en diferentes contextos de uso. Frente a esta disyuntiva se ha incluido en el subsistema de Apreciación la categoría de "Corrección" que no tendría, entonces, el carácter ético que asumen Martin, Rose y White, sino más bien como una evaluación que engloba un carácter de composición, valoración y adecuación con la norma considerada "estándar" o "más educada" por los hablantes.

\section{ANÁLISIS}

4.1. Evaluaciones de los(as) HBs sobre el español en un sentido amplio. Las evaluaciones de los(as) HBs sobre el español consultados(as) en este estudio, en general tienden a expresarse en juicios positivos de Estima Social. La gran mayoría asocia esta evaluación positiva con las posibilidades laborales que el conocimiento del español ofrece en Estados Unidos. Es muy común que además estos juicios de Estima Social positivos vayan acompañados de una fuerza de graduación alta: "muy importante". Las evaluaciones de Afecto están asociadas al español en afirmaciones que expresan que esta lengua es parte de sus vidas, de su cultura y de su identidad. Sin embargo, los(as) HBs incluyen evaluaciones de Afecto negativo al expresar su miedo e inseguridad de perder u olvidar su español, o en relación a no poder mantener sus habilidades de hablarlo coloquialmente o fallar en su uso en contextos que requieren un español más formal y académico. Este sentimiento negativo de miedo e inseguridad se expresa principalmente a través de la polaridad negativa y mediante el uso de verbos mentales: "No quiero perder mi español", "Yo no quiero olvidar mi español", "Me gustaría no olvidar [el español]". Algunos(as) participantes expresan que su baja proficiencia en esta lengua se debe a la falta de oportunidades que tuvieron de practicar español mientras crecían. Los(as) HBs se expresan usando en general un modo monoglósico, con una baja frecuencia de proyecciones mentales y verbales que permitan la inclusión de otras voces en su discurso (las proyecciones son empleadas como se ha manifestado para expresar Afecto negativo de inseguridad). Algunos expresan evaluaciones de Estima Social negativa e incluso de Sanción Social de propiedad (moral, ético, justo) negativa acerca de los padres que no enseñan español a sus hijos, y asimismo de los profesores de enseñanza primaria y secundaria que no permitían a sus estudiantes hablar español en las escuelas públicas².

Los participantes muestran un fuerte sentimiento de identidad con su comunidad que, de acuerdo a ellos(as) mismos(as), debiera estar conectado con el lenguaje. Ya se ha señalado previamente que los(as) HBs que participaron en este estudio no componen en absoluto un grupo homogéneo; sin embargo, como es posible esperar, la mayoría se siente confiado(a) de usar el español en situaciones de habla coloquiales y sólo algunos(as) de ellos(as) piensan que no se pueden comunicar muy bien con sus amigos de habla hispana y familiares.

Los ejemplos incluidos a continuación son representativos del uso de una polaridad negativa en relación a evaluaciones negativas de Afecto (miedo, inseguridad, vergüenza) y evaluaciones negativas de Estima Social de Capacidad y evaluaciones negativas de Apreciación (Corrección). Verbos como "olvidar" y "perder" asociados a expresiones de miedo a la pérdida del idioma tienen una alta frecuencia en la data analizada (ocho ocurrencias del primero y doce del segundo verbo):

(1) Yo no quiero perder mi lenguaje porque perderlo es como perder una parte de mi cultura.

(2) Yo no quiero perder este idioma. Siento que en los dos años en [ciudad de Washington] yo ha perdido mucha práctica y se me olvida las palabras.

\footnotetext{
${ }^{2}$ En un estudio previo que analiza la ideologías sobre el lenguaje en relación al monolingüismo y bilingüismo en los medios de comunicación local de una comunidad de la frontera en el suroeste de Estados Unidos se constató este mismo tipo de evaluaciones negativas de Afecto de insatisfacción y descontento en cuanto al temor y preocupación por la posible pérdida del español en la comunidad (Achugar y Oteíza en preparación).
} 
(3) ...quiero poder hablar y producirlo bien para no tener verguenza.

(4) Tengo mucha familia que por una rason o otra no hablan el español y eso me averguenza mucho [fuerza, graduación alta] porque es la lengua en que sus ancesters hablaron y se acabo con ellos. Yo no quiero que eso pase conmigo.

\section{(5) No me gustaría olvidar.}

El ejemplo (4) nos presenta un caso de heteroglosia por comparación. La participante expresa una valoración de Afecto y de Estima Social negativas de lo que "debería ser" la actitud de su familia, es decir, que ésta debería haber mantenido su español y ella no quiere ser partícipe de esa situación que le provoca mucha vergüenza. A continuación se presentan algunos ejemplos de modalidad positiva asociada con evaluaciones positivas de Estima Social de capacidad (positiva y neutra) y evaluaciones positivas de Afecto en relación a las oportunidades laborales y la confianza en sus capacidades de progresar en el aprendizaje de la lengua al mismo tiempo que expresan una valoración de su bilingüismo:

(6) Mi vida es más fácil porque puedo comunicarme con personas que hablan inglés y español.

(7) Me gustaría poder comunicarme mejor ya que es la única [foco] lengua que puedo comunicarme con ellos [familia].

(8) ...sera necesario saber español para poder tener un trabajo en los Estados Unidos.

(9) Sé que puedo mejorar y hablar mas [fuerza] educadamente [foco].

El ejemplo (7), así como el ejemplo (5) previamente mencionado, enfatizan el deseo de los hablantes de mantener la lengua en la esfera privada de la familia a través del verbo condicional que modaliza.

Un 56,6 por ciento de los HBs usa una modalidad positiva, mientras que un 46,6 por ciento emplea más de una vez una polaridad negativa y modalidad asociada con evaluaciones negativas de Afecto que son señales del temor a perder el español o de una capacidad baja de usar esta lengua incluso en situaciones sociales informales y familiares. Estas evaluaciones de Afecto negativas son codificadas a través de procesos mentales y verbales. Si bien es importante considerar que el miedo a perder el español, implícitamente implica una valoración de Estima Social positiva respecto a esta lengua (evaluación no gramaticalizada o expresada léxicamente), y su rol en el sentimiento de pertenencia a una comunidad e identidad cultural.

A continuación se muestra en la tabla 2 una síntesis de estos resultados ${ }^{3}$.

Tabla 2

Síntesis del Análisis Evaluativo del español en un sentido amplio

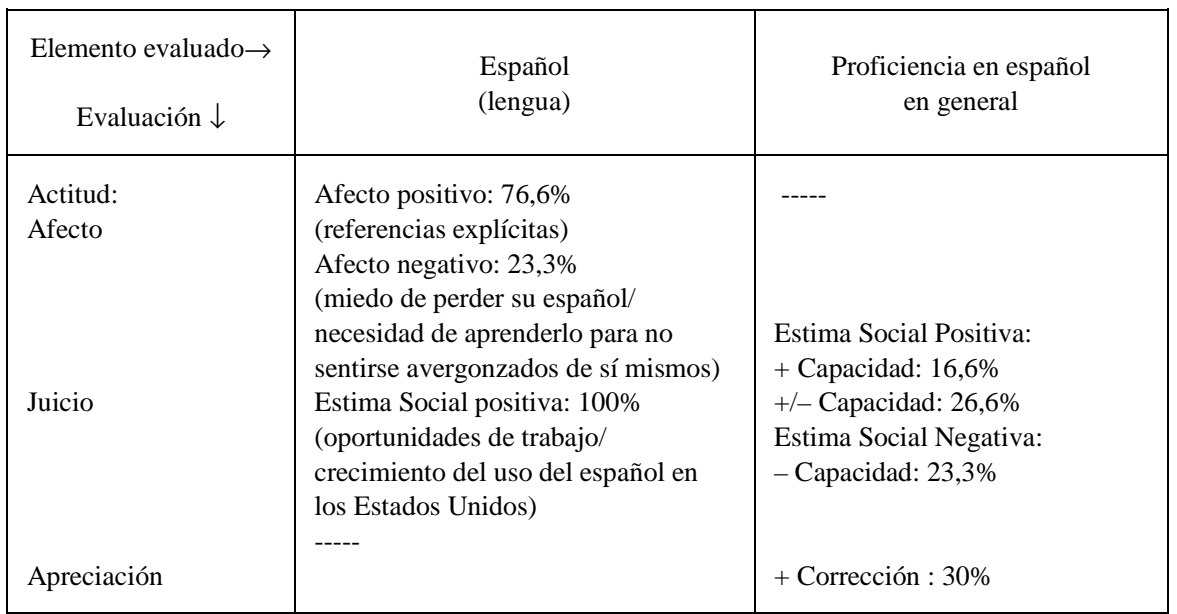

\footnotetext{
${ }^{3}$ La inclusión de porcentajes no pretende convertir este trabajo en un estudio cuantitativo, sino más bien presentar al lector la representatividad de las evaluaciones expresadas por los hablantes. Los porcentajes muestran el número de participantes que expresaron una evaluación determinada, no todos evaluaron de acuerdo a todas las categorías empleadas en el estudio.
} 


\begin{tabular}{|c|c|}
\hline $\begin{array}{l}\text { Compromiso: } \\
\text { Monoglosia } \\
\text { Heteroglosia } \\
\text { (proyección, } \\
\text { modalidad, } \\
\text { concesión) }\end{array}$ & $\begin{array}{l}\text { Heteroglosia indirecta: profesores previos (Cuatro ocurrencias de Estima } \\
\text { Social negativa). } \\
\text { Modalidad positiva: asociada con una evaluación positiva de Apreciación } \\
\text { y de Estima Social Positiva de Capacidad. }\end{array}$ \\
\hline $\begin{array}{l}\text { Graduación: } \\
\text { Fuerza } \\
\text { (graduación alta) }\end{array}$ & $\begin{array}{l}\text { Asociada a evaluaciones positivas de Estima Social: } \\
\text { gran ventaja: } 23,3 \% \\
\text { gran parte/ gran cantidad/ mucha gente/ muchos hispanos... 33,3\% } \\
\text { muy importante: } 56,6 \%\end{array}$ \\
\hline
\end{tabular}

4.2. Evaluaciones de hablantes bilingües (HBs) de su proficiencia en diferentes registros y usos. En esta sección se presentan las percepciones que manifestaron los(as) participantes del estudio, ya no del español en general, sino de su propia proficiencia en diferentes contextos y registros de uso.

Los ejemplos presentados a continuación muestran las percepciones que los(as) HBs manifestaron en relación a su proficiencia oral informal con amigos. En sus evaluaciones se aprecia una polaridad negativa asociada a valoraciones negativas de Apreciación en cuanto al criterio de corrección del uso del español:

(10) Puedo hablar claramente aunque sin duda [concesión, modalización]. hay algunas veces que se que aunque [concesión] nos entendemos no estamos hablando correctamente.

(11) No uso las palabras correctas.

(12) Yo e moldeado mi español hacia un español callejero de esta región.

(13) trato de hablar los mas correcto posible en español...

(14) ... pero prefiero hablar con mis amigos en ingles. Creo que talves es por miedo de ser criticada.

En el ejemplo (10) se aprecia una autocrítica en la que se valora más la "corrección" del idioma por sobre el hecho de comunicarse con éxito. Esta valoración negativa es particularmente representativa de la experiencia de muchos(as) HBs de español en Estados Unidos que comparten la percepción de que su español no responde a una versión estándar. Así también, en el ejemplo (14) vemos que se le da importancia a la opinión de otros (heteroglosia) a través de una valoración de Afecto negativo de inseguridad expresando indirectamente una valoración negativa de la propia producción del hablante.

Parece interesante señalar que un 36,6 por ciento de los participantes comienzan sus oraciones expresando que ellos creen que su capacidad para comunicarse informalmente con amigos en español es "muy buena", si bien la siguiente cláusula se inicia por un marcador discursivo adversativo que restringe esta capacidad (pero/sin embargo/aunque) y marca el inicio de la idea de la "palabra correcta" o simplemente de la concepción general del uso correcto del lenguaje. El empleo de la concesión que genera una expectativa contraria a la que había creado el hablante en la mente del lector responde a la percepción de los(as) HBs de que su uso del lenguaje no es todo lo desarrollado o educado que debiera ser. Esta evaluación de Apreciación negativa de Corrección es comúnmente amplificada por el recurso de la fuerza: "Se me olvidó mucho; Necesito más desarrollo". Esta última idea es expresada por el 50 por ciento de los participantes en este estudio.

Tabla 3

Síntesis de Análisis Evaluativo de la Proficiencia oral informal con amigos

\begin{tabular}{|l|l|}
\hline $\begin{array}{c}\text { Evaluación de } \\
\text { Actitud } \rightarrow\end{array}$ & Actitud: \\
\hline
\end{tabular}




\begin{tabular}{|c|c|c|c|}
\hline Elemento evaluado $\downarrow$ & Afecto & Juicio & Apreciación \\
\hline $\begin{array}{l}\text { Proficiencia oral } \\
\text { (informal, con amigos) }\end{array}$ & $\begin{array}{l}\text { Afecto positivo: } 50 \% \\
\text { Afecto negativo: } 0 \%\end{array}$ & $\begin{array}{l}\text { Estima Social } \\
\text { positiva: } \\
\text { + capacidad: } 36,6 \% \\
\text { [seguida de } \\
\text { concesión] } \\
\text { +/-Capacidad: } 56,6 \% \\
\text { Estima Social } \\
\text { negativa: } \\
\text { - Capacidad: } 6,6 \%\end{array}$ & - Corrección: 50\% \\
\hline
\end{tabular}

En relación a las percepciones sobre su proficiencia oral informal con padres y familiares, las evaluaciones de Apreciación negativas de Corrección son las que poseen el porcentaje más alto. Los ejemplos 15-18 ilustran este tipo de evaluación de los(as) HBs:

(15) Con mis padres nuestro español es muy informal o "mocho" le decimos".

(16) Tenemos tendencias de usar palabras inventadas por la familia o que no existen en español.

(17) Mis amigos que no son familiares son alumnos en el colegio [universidad] que hablan español y con ellos me esfuerzo a hablar el español lo mas correcto posible.

(18) Con mi familia hablamos un español más relajado pero a la misma vez tratamos de no decir palabras erróneas.

(19) Cada familia tiene ciertos nombres [foco] que le han dado a ciertas cosas [foco] a través los anos que se les pega pero que no son palabras correctas.

El uso del "nosotros" hace referencia a ese sentimiento de pertenencia a una comunidad de habla representada por su familia: "nuestro español"/ "decimos" que señala una evidente inclusión de otros interlocutores en el discurso a través de una proyección verbal. Se constata, entonces, la fuerte percepción de que existe una separación entre el lenguaje familiar y cotidiano y la percepción de lo que es correcto como deja de manifiesto el ejemplo (19) o más aún el ejemplo (16) que se refiere a las palabras que "no existen". Todos los testimonios tienen en común el mostrar una conciencia de los(as) HBs de las variaciones del español que son parte del habla en el espacio privado, pero que no corresponden a una lengua estándar. El uso de la concesión para marcar una evaluación de Apreciación negativa de Corrección también está presente en las expresiones sobre la competencia oral informal de los(as) HBs. Una síntesis de estas evaluaciones puede apreciarse en la tabla 4.

Tabla 4

Síntesis de Análisis Evaluativo de la Proficiencia oral informal con padres y familiares

\begin{tabular}{|c|c|c|c|}
\hline & \multicolumn{3}{|c|}{ Actitud: } \\
\hline Elemento evaluado $\downarrow$ & Afecto & Juicio & Apreciación \\
\hline $\begin{array}{l}\text { Proficiencia oral } \\
\text { (informal, con padres } \\
\text { y familiares) }\end{array}$ & $\begin{array}{l}\text { No hay evaluaciones } \\
\text { de Afecto }\end{array}$ & $\begin{array}{l}\text { Estima Social } \\
\text { positiva: } \\
\text { + capacidad: } 50 \%\end{array}$ & - Corrección: 56,6\% \\
\hline
\end{tabular}

\footnotetext{
${ }^{4}$ El término "mocho" en general posee una connotación negativa entre los hablantes bilingüies y no bilingües en Estados Unidos. Esta denominación es dada por los parientes monolingües que viven en México y que consideran que el español de quienes viven en Estados Unidos no responde a lo que ellos perciben como la norma.
} 


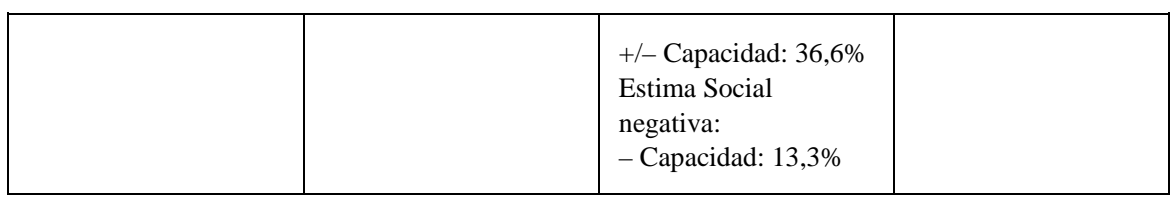

En relación a su capacidad para escribir correos electrónicos en español, los participantes marcaron una diferencia entre escribirle a personas de Estados Unidos y a una persona fuera del país, en especial a México, como se les había solicitado en el cuestionario de percepciones (ver Anexo). Las evaluaciones de Apreciación negativa de Corrección y de Estima Social negativa de Capacidad se refieren a sus percepciones sobre su capacidad para expresarse en español al escribir a personas que viven fuera de Estados Unidos. Ejemplos 20 y 21 son representativos de sus respuestas:

(20) Me encuentro usando mucho [fuerza] el Spanglish.

(21) Al escribir con alguien fuera de aquí por ejemplo mis primos en México necesito saber los acentos y las palabras correctas porque si no las se no puedo decirlas en inglés.

Tabla 5

Síntesis de Análisis Evaluativo de la Proficiencia escrita (correos electrónicos)

\begin{tabular}{|c|l|l|l|}
\hline \multirow{2}{*}{$\begin{array}{c}\text { Evaluación de } \\
\text { Actitud } \rightarrow\end{array}$} & \multicolumn{3}{|c|}{ Actitud: } \\
\cline { 2 - 4 } Elemento evaluado $\downarrow$ & \multicolumn{1}{|c|}{ Afecto } & \multicolumn{1}{|c|}{ Juicio } & Apreciación \\
\hline $\begin{array}{l}\text { Proficiencia escrita } \\
\text { (Español escrito: } \\
\text { correos electrónicos) }\end{array}$ & $\begin{array}{l}\text { No hay evaluaciones } \\
\text { de Afecto }\end{array}$ & $\begin{array}{l}\text { Estima Social } \\
\text { positiva: } \\
+ \text { capacidad: } 43,3 \% \\
+ \text { - Capacidad: } 33,3 \% \\
\text { Estima Social } \\
\text { negativa: } \\
\text { - Capacidad: } 6,6 \%\end{array}$ & - Corrección: 23,3\% \\
\hline
\end{tabular}

En cuanto a las evaluaciones expresadas por los(as) HBs en relación al uso del español formal (o académico) oral o escrito, parece significativo que un alto porcentaje de ellas tengan un carácter afectivo (ver tabla 6) y contrariamente a lo que se podría esperar, el porcentaje de evaluaciones de Apreciación negativa de Corrección es menor. Parece relevante que el 78,5 por ciento de los(as) informantes señala que "Necesito más desarrollo/ práctica" en el uso de la lengua formal sea ésta escrita u oral. Los ejemplos 22-24 a continuación son representativos de sus opiniones:

(22) ... me costó trabajo tratar de eliminar los rasgos dialécticos cuando estaba estudiando el español más formal.

El español que aprendí, obviamente [modalización] reflejaba el modo de hablar de la región de donde venía mi familia en Michoacan, México.

(23) Mi español formal no es tan bueno como yo lo quisiera [modalización].

(24) Me falta mucho [fuerza] por aprender. Realmente [modalización, adjunto comentario] expresandome oralmente [foco] es mi preferencia.

Tabla 6

Síntesis de Análisis Evaluativo de la Proficiencia formal (escrita y oral)

\begin{tabular}{|l|l|}
\hline $\begin{array}{c}\text { Evaluación de } \\
\text { Actitud } \rightarrow\end{array}$ & Actitud: \\
\hline
\end{tabular}




\begin{tabular}{|c|c|c|c|}
\hline Elemento evaluado $\downarrow$ & Afecto & Juicio & Apreciación \\
\hline $\begin{array}{l}\text { Proficiencia formal } \\
\text { Escrita: } \\
\text { Oral: }\end{array}$ & $\begin{array}{l}\text { Afecto positivo: } \\
\text { 13,3\% } \\
\text { Afecto negativo: } 6,6 \% \\
\text { Afecto positivo: } 30 \%\end{array}$ & $\begin{array}{l}\text { Estima Social } \\
\text { positiva: } \\
\text { + capacidad: } 13,3 \% \\
\text { +/- Capacidad: } 56,6 \% \\
\text { Estima Social } \\
\text { negativa: } \\
\text { - Capacidad: } 6,6 \%\end{array}$ & - Corrección: 13,3\% \\
\hline
\end{tabular}

La inclusión de otras voces distintas a los(as) BHs es muy alta en relación a las evaluaciones de su proficiencia formal oral en español manifestando una sensibilidad hacia la audiencia. Algunas de las voces que incluyeron los(as) participantes que colaboran con esta heteroglosia son las siguientes: (i) compañeros de clase: Estima Social positiva de capacidad 35,7 por ciento; (ii) profesores universitarios: Estima Social positiva de capacidad 21,4 por ciento, mediana capacidad: 28,5 por ciento.

Cuatro informantes manifestaron el sentimiento de solidaridad que percibían de sus profesores, el ejemplo (25) es representativo de esta valoración ${ }^{5}$. El ejemplo (26) nos muestra una valoración de Apreciación negativa de Corrección y el ejemplo (27), en cambio, es una expresión de un fuerte Afecto negativo mientras se sigue valorando la participación y solidaridad de los profesores:

(25) Creo que mis compañeros muchas veces [fuerza] no se notan de errores que hago y que por lo tanto me dirán que lo manejo bien, pero [concesión] sé que tengo áreas en que puedo mejorarme y creo los profesores si se dan cuento de eso y agradezco las sugerencias que me dan para poder hacerlo.

(26) Cuando tengo que expresarme en español en una presentación formal trato lo mas posible de usar palabras y expresiones que se decir o usar correctamente.

(27) Mis profesores en mas de una ocasión me han ayudado durante la presentación, se que ellos sienten y han visto la frustración que tengo cuando busco las palabras correctas para expresarme bien.

El ejemplo (28) refleja una evaluación negativa de Afecto (culpa) a través del empleo de la modalidad negativa. Nótese el uso de "especialmente"/ "por lo menos" (foco) que hace más enfática la culpabilidad del participante y la asignación de responsabilidad que recae únicamente en sí mismo y el fuerte sentimiento de lo que "debiera ser" su capacidad de expresarse en su lengua nativa o de toda la vida. Por otro lado, el ejemplo (29) que fue entregado por una HB deja entrever, al igual que el HB de ejemplo (28), el "deber ser" o la obligación que sienten muchos(as) HBs de "hablar bien" porque consideran el español su lengua nativa, sin tomar en cuenta que los registros académicos requieren de una práctica y formación formal que implica el manejo de determinadas demandas lingüísticas de estructuración del lenguaje que difieren de la lengua usada en ambientes familiares. Las evaluaciones de Afecto negativo, de inseguridad en relación a su capacidad para expresarse oral y formalmente en español, reciben un alto porcentaje que sobrepasa ampliamente sus valoraciones de Juicio (Estima Social de Capacidad). Estas evaluaciones pueden apreciarse en la tabla 7.

(28) ...pero me siento mal porque ellos me han enseñado bien y yo debería de saber por lo menos como hablar en español especialmente [foco] cuando soy un nativo de un país español.

(29) Pienso que cuando presente dentro de classe mis compañeros al igual que la profesora seran severos con al evaluación porque saben que hablo español de toda la vida.

Tabla 7

Síntesis de Análisis Evaluativo de la Proficiencia formal oral

\footnotetext{
${ }^{5}$ Es necesario tener en cuenta en el momento de evaluar estas respuestas que los HBs tienen conciencia de que la investigadora es a la vez profesora de español de la universidad.
} 


\begin{tabular}{|l|l|l|l|}
\hline \multirow{2}{*}{$\begin{array}{c}\text { Evaluación de } \\
\text { Actitud } \rightarrow\end{array}$} & \multicolumn{2}{|c|}{ Actitud: } & \multicolumn{2}{|c|}{ Apreciación } \\
\cline { 2 - 4 } Elemento evaluado $\downarrow$ & \multicolumn{1}{|c|}{ Afecto } & \multicolumn{1}{|c|}{ Juicio } \\
\hline $\begin{array}{l}\text { Español oral formal } \\
\text { (presentación enfrente } \\
\text { de una clase de } \\
\text { lengua, literatura o } \\
\text { cultura) }\end{array}$ & $\begin{array}{l}\text { Afecto positivo: } 6,6 \% \\
\text { Afecto negativo: } 50 \% \\
\text { Inseguridad, Algunos } \\
\text { de ellos a través de } \\
\text { evaluaciones muy } \\
\text { fuertes) }\end{array}$ & $\begin{array}{l}\text { Estima Social } \\
\text { positiva: } \\
+ \text { capacidad: } 33,3 \% \\
+/- \text { Capacidad: } 30 \% \\
\text { Estima Social } \\
\text { negativa: } \\
\text { - Capacidad: } 30 \%\end{array}$ & - Corrección: 42,8\% \\
\hline
\end{tabular}

Por último, en cuanto a su capacidad para expresarse formalmente por escrito, los(as) participantes de este estudio manifestaron sentirse medianamente competentes o con muy baja capacidad, sólo un 13,3 por ciento expresó seguridad en su escritura. Es notorio que el mayor porcentaje de sus evaluaciones haya sido expresado en términos de Afecto negativo (70\%).

El ejemplo (30) nos muestra una fuerte evaluación de Capacidad negativa (Estima Social) en relación a la proficiencia formal escrita que expresa cómo frente a la dificultad de la tarea, el participante se rinde en el transcurso del proceso:

(30) El proceso de encontrar la información es fácil, pero cuando llega el proceso de desarrollar esa información en mis propias palabras me quiebro la cabeza, [evaluación de capacidad negativa indirecta] pasan horas y horas escribiendo y borrando varias frases asta que al fin uso palabras simples para simplemente [foco] terminar la tarea.

(31) Pienso que mi capacidad para escribir trabajos formalmente [foco] en español es bajo.

En la tabla 8 es posible apreciar una síntesis que sólo abarca el español escrito y formal:

Tabla 8

Síntesis de Análisis Evaluativo de la Proficiencia formal escrita

\begin{tabular}{|c|c|c|c|}
\hline Evaluación de & \multicolumn{3}{|c|}{ Actitud: } \\
\hline Elemento evaluado $\downarrow$ & Afecto & Juicio & Apreciación \\
\hline Español escrito formal & $\begin{array}{l}\text { Afecto positivo: } 6,6 \% \\
\text { Afecto negativo: } 70 \%\end{array}$ & $\begin{array}{l}\text { Estima Social } \\
\text { positiva: } \\
\text { + capacidad: } 13,3 \% \\
\text { +/- Capacidad: } 50 \% \\
\text { Estima Social } \\
\text { negativa: } \\
\text { - Capacidad: } 30 \%\end{array}$ & $\begin{array}{l}\text { No hay evaluaciones } \\
\text { de Corrección, }\end{array}$ \\
\hline
\end{tabular}

\section{CONCLUSIONES}

Los(as) HBs que formaron parte de este estudio consideran que su identidad se construye en parte a través de su habilidad para producir en español, tanto oral como escrito. El 23,3 por ciento de los(as) HBs expresan una inseguridad en relación al español (evaluación de Afecto negativo) manifestando su temor de perder su competencia en este lenguaje. Otros expresan su inseguridad de usar el español en determinadas oportunidades, con sus parientes o con hablantes que ellos consideran más proficientes en la lengua. Indudablemente que las expresiones de Afecto negativo de inseguridad se manifiestan con 
más fuerza en sus evaluaciones del español formal oral y escrito (50 y 70 por ciento respectivamente).

Este grupo de HBs manifiesta una evaluación negativa de su proficiencia en español en ambientes más formales o académicos y tienden a pensar que el ser proficiente en español y tener el nivel de lenguaje que necesitan para desenvolverse con éxito en otros contextos que no sean las interacciones coloquiales con amigos hispanos y parientes, es su propia responsabilidad. Asimismo, tienden a pensar que porque tienen un fondo de habla hispana, y porque pueden hablar español, debieran ser capaces de usar el lenguaje en otros registros (registros académicos) con más facilidad que otros estudiantes que han aprendido el español como segunda lengua, aunque no hayan recibido una educación formal en esta lengua. Las percepciones de los(as) HBs en cuanto a su proficiencia en registros académicos o más formales se relacionan con el uso "correcto o incorrecto" del léxico y de la ortografía, reproduciendo de este modo, un discurso de la "corrección" o del "deber ser del lenguaje". Ellos perciben su propio progreso en el español en general como una necesidad para evitar usar sus propias variantes dialectales informales del español e incorporar un uso más "educado" o "estándar" de esta lengua, y, así, expresarse con un español "menos callejero", "eliminar" usos incorrectos o no incluir "palabras que no existen".

Si los(as) HBs enfrentados(as) al desafío que significa aprender el español en contextos más formales tienden a responsabilizarse a sí mismos(as), faltaría desarrollar una conciencia, tanto en profesores como en ellos/ellas mismos(as), de que la adquisición y desarrollo de registros académicos en cualquier lengua requiere de una instrucción y práctica formal, sistemática y especializada, y que esto es aún más crucial en el caso de lenguas minoritarias como el español inserto en un país en el que el inglés es el idioma dominante y de prestigio.

Pareciera necesario además estimular la discusión sobre qué se entiende por un "lenguaje estándar" en el español escrito y formal en Estados Unidos, y generar más investigación en relación a los valores simbólicos que reciben los diferentes dialectos del español empleado dentro y fuera de Estados Unidos como una herramienta de identificación y pertenencia a determinados grupos sociales. En esta línea, Daniel Villa (2000) enfatiza el aspecto pluricéntrico del español dado los múltiples centros que proyectan normas de lo que se considera correcto en el uso del lenguaje; él señala que "es extremadamente difícil de determinar si el español de Estados Unidos es estándar o no dada la total falta de investigación acerca de lo que podría ser un español estándar" (2000: 151).

Finalmente, si pensamos que los significados son realizados al interior de las comunidades parece relevante conectar los discursos particulares con los discursos que representan los hábitos de habla sociales y el accionar de una comunidad como un todo.

\section{OBRAS CITADAS}

Acevedo, Rebeca. 2003. "Navegando a través del registro formal. Curso para hispanohablantes bilingües". En Roca, Ana y Cecilia Colombi (eds.). Mi lengua. Spanish as a heritage language in the United States. Washington, D.C.: Georgetown University Press.

Achugar, Mariana. 2003. "Academic Registers in Spanish in the U.S. A Study of Oral Texts Produced by Bilingual Speakers in a University Graduate Program". En Roca, Ana y Cecilia Colombi (eds.). Mi lengua. Spanish as a heritage language in the United States. Washington, D.C.: Georgetown University Press.

Achugar, Mariana. 2007. "Counter-hegemonic language practices and ideologies: creating a new space and value for Spanish in Southwest Texas" (en prensa).

Achugar, Mariana y Teresa Oteíza. 2007. "In whatever language people feel confortable: conflicting language ideologies in the U.S. Southwest border" (en proceso).

Beckstead, Karen and Almeida J. Toribio. 2003. "Minority Perspectives on Language. Mexican and MexicanAmerican Adolescents' Attitudes toward Spanish and English”. En Roca, Ana y Cecilia Colombi (eds.). Mi lengua. Spanish as a heritage language in the United States. Washington, D.C.: Georgetown University Press.

Carrasco, Roberto Luis y Florencia Riegelhaupt. 2003. "META: A Model for the Continued Acquisition of Spanish by Spanish/English Bilinguals in the United States". En Roca, Ana y Cecilia Colombi (eds.). Mi lengua. Spanish as a heritage language in the United States. Washington, D.C.: Georgetown University Press.

Castillo, Jenny M. 2003. "Teaching Spanish to Heritage Speakers: Issues of Language and Identity”, Geolinguistics.

Colombi, Cecilia. 2000. "En vías del desarrollo del lenguaje académico en español en hablantes nativos de español en los Estados Unidos". En Roca, Ana (ed.). Research on Spanish in the United States: Linguistics Issues and Challenges. Somerville, MA: Cascadilla Press.

—. 2003. "Un enfoque funcional para la enseñanza del ensayo expositivo". En Roca, Ana y Cecilia Colombi (eds.). Mi lengua. Spanish as a heritage language in the United States. Washington, D.C.: Georgetown 


\section{University Press.}

Colombi, Cecilia and Mary J. Schppegrel. 2002. "Theory and Practice in the Development of Advanced Literacy". En Schppegrell, Mary J. y Cecilia Colombi (eds.). Developing Advanced Literacy in First and Second Languages. London: Lawrence Erlbaum Associates, Publishers.

Christie, Frances. 1999. Pedagogy and the Shaping of Consciousness: Linguistics and Social Processes. London: Continuum.

Eggins, Suzanne. 1994. An Introduction to Systemic Functional Linguistics. London: Pinter Publishers.

Eggins, Suzanne y J.R. Martin. 1997. "Genres and Registers of Discourse". En van Dijk, Theun (ed.). Discourse as Structure and Process. London: SAGE Publications, Vol I.

Halliday, M.A.K. 1994. An Introduction to Functional Grammar. London: Edward Arnold.

Halliday, M.A.K. 2001. "Literacy and Linguistics: Relationships between spoken and written language". En Burns, Anne y Caroline Coffin (eds.). Analysing English in a Global Context. London and New York: Routledge.

Hunston, Susan y Geoff $\square$ Thompson (eds.). 2000. Evaluation in Text. Oxford: Oxford University Press.

Lipski, John M. 2000. "Back to Zero or Ahead to 2001? Issues and Challenges in U.S. Spanish Research". En Roca, Ana (ed.). Research on Spanish in the United States: Linguistics Issues and Challenges. Somerville, MA: Cascadilla Press.

Martin, J.R. 2000. "Beyond Exchange: APPRAISAL Systems in English”. En Hunston, Susan y Geoff Thompson (eds.). Evaluation in Text. Oxford: Oxford University Press.

Martin, J.R. \& David Rose. 2003. Working with Discourse. Meaning Beyond the clause. London: Continuum.

Martin, J.R. y P. White. 2005. The Language of Evaluation. Appraisal in English. New York: Palgrave Macmillan.

Oteíza, Teresa. 2007. (En prensa). “Acquiring Spanish Academic Language in the U.S.: A Study of Undergraduate Bilingual Heritage Speakers".

Potowki, Kim. 2002. "Experiences of Spanish Heritage Speakers in University Foreign Language Courses and Implications for Teacher Training". ADFL Bulletin Vol. 33. 3: 35-42.

Silva-Corvalán, Carmen. 1994. Language Contact and Change: Spanish in Los Angeles. New York: Oxford University Press.

Silva-Corvalán, Carmen. 2001. Sociolingüística y pragmática del español. Washington, DC: Georgetown University Press.

Schleppegrell, Mary. 2004. The Language of Schooling. A Functional Linguistics Perspective. London: Lawrence Erlbaum Associates, Publishers.

Valdés, Guadalupe and Michelle Geoffrion-Vinci. 1998. "Chicano Spanish: The Problem of the 'Underdeveloped' Code in Bilingual Repertoires". The Modern Language Journal 82(iv): 473-501.

Villa, Daniel. 2000. "Languages have armies, and economies, too: the presence of U.S. Spanish in the SpanishSpeaking World”. Southwest Journal of Linguistics 19, 2: 143-154. 